Bull. Austral. Math. Soc.

VOL. 68 (2003) [221-231]

\title{
LINEAR MAPS LEAVING INVARIANT SUBSETS OF NONNEGATIVE SYMMETRIC MATRICES
}

\author{
Hanley Chiang and Chi-Kwong Li
}

\begin{abstract}
Let $\mathcal{S}$ be a certain set of nonnegative symmetric matrices, such as the set of symmetric doubly stochastic matrices or the set, of symmetric permutation matrices. It is proven that a linear transformation mapping $\mathcal{S}$ onto $\mathcal{S}$ must be of the form $X \mapsto P^{t} X P$ for some permutation matrix $P$ except for several low dimensional cases.
\end{abstract}

\section{INTRODUCTION}

There has been considerable interest in studying linear transformations on matrix spaces leaving a certain subset invariant, that is, mapping the subset onto itself; see [4]. For example, if $\mathcal{S}$ is the set of $n \times n$ doubly (sub-)stochastic matrices or the set of $n \times n$ (sub-)permutation matrices, and $\mathcal{V}=\operatorname{span} \mathcal{S}$ is the linear span of $\mathcal{S}$, then a linear transformation $\phi: \mathcal{V} \rightarrow \mathcal{V}$ satisfying $\phi(\mathcal{S})=\mathcal{S}$ must be of the form

$$
X \mapsto P X Q \quad \text { or } \quad X \mapsto P X^{t} Q
$$

for some $n \times n$ permutation matrices $P$ and $Q$. In [1], the authors of this paper solved the open problem in [4] concerning linear transformations on span $\mathcal{S}$ satisfying $\phi(\mathcal{S})=\mathcal{S}$, where $\mathcal{S}$ is the set of $n \times n$ even permutation matrices, that is, permutation matrices with determinant one. It was shown that for $n \geqslant 5$, the transformation has the form (1) for some permutation matrices $P, Q$ such that $\operatorname{det}(P Q)=1$. When $n \leqslant 4$, there may be other types of transformations and they are also characterised.

In [5], the authors studied linear transformations $\phi$ satisfying $\phi(\mathcal{S})=\mathcal{S}$, where $\mathcal{S}$ is the set of symmetric doubly stochastic matrices or the set of symmetric doubly substochastic matrices. It was shown that such transformations have the form

$$
X \mapsto P^{t} X P
$$

for some permutation matrix $P$. The analysis in [5] depends on some intricate graph theory. In this paper, we give a short, direct proof for this result, and we study linear maps leaving invariant other matrix sets $\mathcal{S}$ or their convex hulls, where $\mathcal{S}$ is

Received 14th January, 2003

Research supported by an NSF grant.

Copyright Clearance Centre, Inc. Serial-fee code: 0004-9727/03 \$A2.00+0.00. 
$\mathbf{S P}_{n}$ : the set of $n \times n$ symmetric permutation matrices; or

$\mathbf{T}_{n}$ : the set of $n \times n$ permutation matrices corresponding to the identity or transpositions, that is, 2-cycles.

The following notations will also be used in our discussion:

$\mathbf{M}_{n}$ : the set of $n \times n$ real matrices;

$\mathrm{SDS}_{n}$ : the set of $n \times n$ symmetric doubly stochastic matrices;

$\mathbf{P}_{n}$ : the set of $n \times n$ permutation matrices;

$\mathbf{E}_{n}$ : the set of extreme points of $\mathbf{S D S}_{n}$;

$\mathrm{V}_{n}$ : the set of symmetric matrices in $\mathrm{M}_{n}$ with equal row sums and column sums;

$I_{n}$ : the identity matrix in $\mathbf{M}_{n}$;

$J_{n}$ : the matrix in $\mathbf{M}_{n}$ with all entries equal to one;

$T_{i j}$ : the matrix obtained from $I_{n}$ by interchanging the $i$ th and $j$ th rows;

$\tilde{J}_{n}=J_{n}-I_{n}$.

Evidently we have

$$
\mathbf{T}_{n}=\left\{I_{n}\right\} \cup\left\{T_{i j}: 1 \leqslant i<j \leqslant n\right\} .
$$

Moreover, we have the following description of $\mathbf{E}_{n}$ (see $[2,3]$ ).

PROPOSITION 1.1. The set $\mathbf{E}_{n}$ consists of matrices in $\mathbf{M}_{n}$ that are permutationally similar to the direct sums of matrices of the following forms:

(a) The $1 \times 1$ matrix (1);

(b) The $2 \times 2$ matrix $\left(\begin{array}{ll}0 & 1 \\ 1 & 0\end{array}\right)$;

(c) The $k \times k$ symmetric matrix with $1 / 2$ at its $(r, s)$ position with $\{r, s\}$ $=\{1, k\}$ or $\{r, s\}=\{i, i+1\}$ for $i=1, \ldots, k-1$, and with zero elsewhere, where $k$ is an odd integer $\geqslant 3$.

Clearly, for a compact convex set $\mathcal{K}$, a linear map $\phi$ leaves $\mathcal{K}$ invariant if and only if $\phi$ leaves invariant the set of extreme points of $\mathcal{K}$. Since $\mathcal{S}$ is the set of extreme points of conv $\mathcal{S}$ for $\mathcal{S}=\mathbf{T}_{n}, \mathbf{S P}_{n}$ and $\mathbf{E}_{n}$, we see that a linear operator $\phi$ satisfies $\phi(\mathcal{S})=\mathcal{S}$ if and only if $\phi(\operatorname{conv} \mathcal{S})=\operatorname{conv} \mathcal{S}$.

We shall present some preliminary results and characterise those linear maps that leave invariant $\mathbf{T}_{n}$ or conv $\mathbf{T}_{n}$ in Section 2. We then characterise those linear maps that leave invariant $\mathbf{S P}_{n}$ or conv $\mathbf{S P}_{n}$ in Section 3 and treat the problem for $\mathbf{E}_{n}$ or $\mathbf{S D S}_{n}$ in Section 4.

We thank Professor Bit-Shun Tam for some helpful comments on an earlier draft of our paper and for showing us the preprint [5]. 


\section{Preliminary Results}

Proposition 2.1. The set $T_{n}$ is a basis for $V_{n}$. Consequently, there is a one-one correspondence between bijections from $\mathbf{T}_{n}$ to $\mathbf{T}_{n}$ and linear maps $\phi: \mathbf{V}_{n} \rightarrow \mathbf{V}_{n}$ satisfying $\phi(\mathcal{S})=\mathcal{S}$ for $\mathcal{S}=\mathbf{T}_{n}$ or conv $\mathbf{T}_{n}$.

PROOF: Recall that $T_{i j}$ is the matrix obtained from $I_{n}$ by interchanging the $i$ th and $j$ th rows, that is, $T_{i j}$ corresponding to the transposition permutation that interchanges $i$ and $j$. If $A=\left(a_{i j}\right) \in \mathbf{V}_{n}$, then

$$
A=\sum_{1 \leqslant i<j \leqslant n} a_{i j} T_{i j}+\mu I
$$

for some suitable $\mu \in \mathbb{R}$ so that the row sums and column sums on both sides match. Thus, $A \in \operatorname{span} \mathrm{T}_{n}$. Also, if $\sum_{i, j} b_{i j} T_{i j}+b_{0} I=0$, then all $b_{i j}=0$ and so must $b_{0}$. Hence, $T_{n}$ is a linearly independent generating set of $\mathbf{V}_{n}$, that is, $\mathbf{T}_{n}$ is a basis for $\mathbf{V}_{n}$.

The last assertion is clear.

CoROLlaRY 2.2. Let $\mathcal{S}$ be any of the sets: $\mathbf{E}_{n}, \mathbf{S P}{ }_{n}, \mathbf{T}_{n}$, conv $\mathbf{E}_{n}$, conv $\mathbf{S P}_{n}$, $\operatorname{conv} \mathrm{T}_{n}$. Then $\operatorname{span} \mathcal{S}=\mathrm{V}_{n}$.

ProOF: Clearly, the linear span of a set is the same as the linear span of the convex hull of the set. We focus on $\mathcal{S}=\mathbf{E}_{n}, \mathbf{S P} \mathbf{P}_{n}$, or $\mathbf{T}_{n}$. Evidently, we have $\mathbf{T}_{n} \subseteq \mathbf{S P} \mathbf{P}_{n} \subseteq \mathbf{E}_{n}$ $\subseteq \mathrm{V}_{n}$, and hence

$$
\operatorname{span} \mathbf{T}_{n} \subseteq \operatorname{span} \mathbf{S P}_{n} \subseteq \operatorname{span} \mathrm{E}_{n} \subseteq \mathrm{V}_{n}
$$

By Proposition 2.1, we have span $T_{n}=V_{n}$. The result follows.

We need the following well-known result in our discussion. We give a short proof for completeness.

Lemma 2.3. If $X \in \mathbf{M}_{n}$ satisfies $P^{t} X P=X$ for all $P \in \mathbf{P}_{n}$, then $X=r I_{n}+s \widetilde{J}_{n}$ for some real numbers $r$ and $s$.

Proof: Let $X=\left(x_{i j}\right)$ satisfy $P^{t} X P=X$ for all $P \in \mathrm{P}_{n}$. All the diagonal entries of $X$ must be equal; otherwise, if $x_{i i} \neq x_{j j}$ for some $i \neq j$, then let $P=T_{i j}$ be the permutation matrix which interchanges rows $i$ and $j$ of $I_{n}$, and it would follow that $P^{t} X P \neq X$. Now, all the off-diagonal entries in the same row of $\mathrm{X}$ must be equal; otherwise, if $x_{i j} \neq x_{i k}$ are two off-diagonal entries in the same row, then let $P=T_{j k}$, and it would follow that $P^{t} X P \neq X$. By a similar argument, all of the off-diagonal entries in the same column of $X$ must be equal. Finally, for any two off-diagonal entries $x_{i j}$ and $x_{k l}$, we have $x_{i j}=x_{k j}=x_{k l}$.

Lemma 2.4. Let $\mathcal{S}=\mathrm{SP}_{n}$ or $\mathbf{E}_{n}$. Then a matrix $A \in \mathcal{S}$ is actually in $\mathrm{T}_{n}$ if and only if

$$
\left\{I_{n}, A\right\}=\left\{X \in \mathcal{S}:\left(I_{n}+2 A-X\right) / 2 \in \operatorname{conv} \mathcal{S}\right\} .
$$

Consequently, if $\phi: \mathrm{V}_{n} \rightarrow \mathrm{V}_{n}$ satisfies $\phi(\mathcal{S})=\mathcal{S}$ and $\phi\left(I_{n}\right)=I_{n}$, then $\phi\left(\mathrm{T}_{n}\right)=\mathrm{T}_{n}$. 
Proof: $(\Rightarrow)$ Suppose $A=I_{n}$. Then clearly $X=I_{n}$ is the only matrix in $\mathcal{S}$ such that $\left(I_{n}+2 A-X\right) / 2 \in \operatorname{conv} \mathcal{S}$. Suppose $A=T_{i j}$, the matrix that corresponds to the transposition interchanging $i$ and $j$. If $X \in \mathcal{S}$ satisfies $\left(I_{n}+2 A-X\right) / 2 \in \operatorname{conv} \mathcal{S}$, then $X$ can only have nonzero entries at the diagonal positions and the $(i, j)$ and $(j, i)$ positions. Thus, $X=I_{n}$ or $T_{i j}$.

$(\Leftarrow)$ Suppose $\mathcal{S}=\mathbf{E}_{n}$ and $A \in \mathcal{S}$ has a $k \times k$ submatrix in rows and columns $i_{1}, \ldots, i_{k}$ such that $k \geqslant 3$ is odd and the $(r, s)$ entry of $A$ is $1 / 2$, where $\{r, s\}=\left\{i_{k}, i_{1}\right\}$ or $\{r, s\}=\left\{i_{j}, i_{j+1}\right\}$ with $j=1, \ldots, k-1$. Then $\left(I_{n}+2 A-X\right) / 2 \in \operatorname{SDS}_{n}$ for any $X=T_{r s}$ with $(r, s)=\left(i_{j}, i_{j+1}\right)$ or $\left(i_{k}, i_{1}\right)$. Next, suppose $\mathcal{S}=\mathbf{E}_{n}$ or $\mathbf{S P}_{n}$ and $A \in \mathcal{S}$ satisfies $A \in \mathbf{S P}_{n} \backslash \mathbf{T}_{n}$. Then there exists $T_{i j} \in \mathbf{T}_{n}$ not equal to $I_{n}$ or $A$ such that $I_{n}+A-T_{i j}=Y \in \mathbf{S P}_{n}$. Thus, $\left(I_{n}+2 A-T_{i j}\right) / 2=(A+Y) / 2 \in \operatorname{conv} \mathbf{S P}_{n} \subseteq$ conv $\mathcal{S}$.

The last assertion is clear.

\section{Symmetric Permutation Matrices}

In this section, we characterise linear maps leaving invariant the sets $\mathbf{S P}_{n}$ or conv $\mathbf{S} \mathbf{P}_{n}$. Since $\mathbf{S} \mathbf{P}_{n}=\mathbf{T}_{n}$ for $n \leqslant 3$, we exclude these cases in our theorem. Note that $T_{p q}, T_{r s} \in \mathbf{T}_{n}$ correspond to disjoint transpositions if and only if $p, q, r, s$ are all distinct.

THEOREM 3.1. Let $\mathcal{S}=\mathbf{S P}_{n}$ or its convex hull. For $n \geqslant 5$, a linear map $\phi: \mathrm{V}_{n} \rightarrow \mathrm{V}_{n}$ satisfies $\phi(\mathcal{S})=\mathcal{S}$ if and only if there is $P \in \mathbf{P}_{n}$ such that $\phi$ has the form $X \mapsto P^{t} X P$. For $n=4$, a linear map $\phi: \mathrm{V}_{4} \rightarrow \mathrm{V}_{4}$ satisfies $\phi(\mathcal{S})=\mathcal{S}$ if and only if $\phi\left(I_{4}\right)=I_{4}$ and $\phi$ permutes the sets $\left\{T_{12}, T_{34}\right\},\left\{T_{13}, T_{24}\right\}$, and $\left\{T_{14}, T_{23}\right\}$.

We establish several lemmas to prove Theorem 3.1. We begin with the following well-known result. A proof is given for completeness.

Lemma 3.2. There exist $A_{1}, \ldots, A_{n} \in \mathbf{S P}_{n}$ such that $A_{1}+\cdots+A_{n}=J_{n}$. Consequently, the matrix $J_{n} / n \in \operatorname{conv} \mathrm{SP}_{n}$.

PROOF: Recall that the complete graph $K_{n}$ is the graph with $n$ vertices such that there is an edge connecting any two vertices. Depict the vertices $1, \ldots, n$ of $K_{n}$ as the points $1, e^{2 \pi / n}, \ldots, e^{i 2(n-1) \pi / n}$ on the complex plane and its edges as straight line segments.

Suppose $n=2 k+1$ is odd. We identify every matrix in $\mathbf{S P}_{n}$ as a permutation, and express the matrix in terms of its cycle decompositions (as a permutation). Let $A_{1}=(1)(2, n)(3, n-1) \cdots(k+1, k+2) \in \mathbf{S P}_{n}$ correspond to the loop at vertex 1 together with the collection of the $k$ edges in $K_{n}$ that are parallel to the edge joining vertices 2 and $n$. Let $A_{2}=(2)(3,1)(4, n)(5, n-1) \cdots(k+2, k+3) \in \mathbf{S P}_{n}$ correspond to the loop at vertex 2 together with the collection of the $k$ edges in $K_{n}$ that are parallel to the edge joining vertices 3 and 1 . Similarly, for $j=3, \ldots, n-1$, let $A_{j}=(j)(j$ $+1, j-1) \cdots \in \mathbf{S P}_{\boldsymbol{n}}$ correspond to the loop at vertex $j$ together with the collection of the $k$ edges in $K_{n}$ that are parallel to the edge joining vertices $j+1$ and $j-1$. Finally, 
let $A_{n}=(n)(1, n-1)(2, n-2) \cdots(k, k+1) \in \mathbf{S P}_{n}$ correspond to the loop at vertex $n$ together with the collection of the $k$ edges in $K_{n}$ that are parallel to the edge joining vertices 1 and $n-1$. One readily checks that $J_{n}=A_{1}+\cdots+A_{n}$.

We extend the above construction to the case when $n+1=2 k+2$ is even as follows. We construct $\widetilde{A}_{i}$ from the $A_{i}$ in the preceding paragraph by replacing $(i)$ with $(i, n+1)$. For example, $\widetilde{A}_{\mathbf{1}}=(1, n+1)(2, n)(3, n-1) \cdots(k+1, k+2) \in \mathbf{S P}_{n+1}$. Then $J_{n+1}=\widetilde{A}_{1}+\cdots+\widetilde{A}_{n}+I_{n+1}$.

LEMMA 3.3. Let $F_{n}$ be the sum of matrices in $\mathbf{S P}_{n}$. Then $F_{n}=f_{n-1} I_{n}+f_{n-2} \widetilde{J}_{n}$, where $f_{k}=\left|\mathbf{S P}_{k}\right|$.

(a) We have $f_{1}=1, f_{2}=2, f_{3}=4$, and $f_{k}=f_{k-1}+(k-1) f_{k-2}>2 f_{k-1}$ for $k \geqslant 4$.

(b) Let $g_{n}=f_{n-1}-f_{n-2}$. For $n \geqslant 5$, a matrix $A \in \mathbf{S P}_{n}$ is the identity matrix if and only if

$$
\left(F_{n}-g_{n} A\right) /\left(f_{n}-g_{n}\right) \in \operatorname{conv} \mathbf{S P}_{n} .
$$

Proof: For any $P \in \mathbf{P}_{n}$, we have

$$
P^{t} F_{n} P=P^{t}\left(\sum_{A \in \mathbf{S P}_{\mathrm{n}}} A\right) P=\sum_{A \in \mathbf{S P}_{n}} P^{t} A P=\sum_{A \in \mathbf{S P}_{\mathbf{n}}} A=F_{n} .
$$

By Lemma $2.3, F_{n}$ is a linear combination of $I_{n}$ and $\widetilde{J}_{n}$. Clearly, there are $f_{n-1}$ matrices in $\mathrm{SP}_{n}$ with 1 at the $(1,1)$ entry, and there are $f_{n-2}$ matrices in $\mathbf{S P}_{n}$ with 1 at the $(1,2)$ entry. Thus, $F_{n}=f_{n-1} I_{n}+f_{n-2} \widetilde{J}_{n}$.

(a) We have $f_{n}=f_{n-1}+(n-1) f_{n-2}$ because both sides of this equation are equal to the common row sum of $F_{n}$. Now, it is easy to check that $f_{1}=1, f_{2}=2, f_{3}=4$, $f_{4}=10, f_{5}=26$. So, $f_{5}>2 f_{4}>4 f_{3}$. If $f_{k}>2 f_{k-1}>4 f_{k-2}$ for some $k \geqslant 5$, then

$$
f_{k+1}-2 f_{k}=f_{k}+k f_{k-1}-2\left(f_{k-1}+(k-1) f_{k-2}\right)>0 .
$$

By the principle of induction, the last assertion follows.

(b) Suppose $n \geqslant 5$. If $A=I_{n}$, then $\left(F_{n}-g_{n} A\right) /\left(f_{n}-g_{n}\right)=J_{n} / n \in \operatorname{conv} \mathbf{S P}_{n}$. If $A$ has an off-diagonal entry equal to one, then $F_{n}-g_{n} A$ has an off-diagonal entry equal to $f_{n-2}-g_{n}=2 f_{n-2}-f_{n-1}<0$, and thus $\left(F_{n}-g_{n} A\right) /\left(f_{n}-g_{n}\right) \notin$ conv $\mathbf{S P}_{n}$.

LEMMA 3.4. Let $n \geqslant 4$. If $\phi: \mathrm{V}_{n} \rightarrow \mathrm{V}_{n}$ satisfies $\phi(\mathcal{S})=\mathcal{S}$, where $\mathcal{S}=\mathrm{SP}_{n}$ or conv $\mathrm{SP}_{n}$, then $\phi\left(F_{n}\right)=F_{n}$ and $\phi\left(I_{n}\right)=I_{n}$.

Proof: Define $F_{n}$ as in Lemma 3.3. Since $\phi\left(\mathbf{S P}_{n}\right)=\mathbf{S P}_{n}$, clearly $\phi\left(F_{n}\right)=F_{n}$. For $n \geqslant 5$, if $\phi\left(I_{n}\right)=A$, then $\left(F_{n}-g_{n} A\right) /\left(f_{n}-g_{n}\right)=\phi\left(\left(F_{n}-g_{n} I_{n}\right) /\left(f_{n}-g_{n}\right)\right)=\phi\left(J_{n} / n\right)$ $\epsilon$ conv SP $_{n}$. By Lemma 3.3(b), we see that $A=I_{n}$.

For $n=4$, let $B_{1}, B_{2}, B_{3}$ be the three elements in $\mathbf{S P}_{4} \backslash \mathbf{T}_{4}$. Then $B_{1}+B_{2}+B_{3}=\widetilde{J}_{4}$, and thus $\widetilde{J}_{4} / 3 \in$ conv $\mathrm{SP}_{4}$. Since $F_{4}=4 I_{4}+2 \widetilde{J}_{4}$, it follows that a matrix $A \in \mathrm{SP}_{4}$ satisfies $\left(F_{4}-4 A\right) / 6 \in$ conv $\mathrm{SP}_{4}$ if and only if $A=I_{4}$. By arguments similar to those in the last paragraph, we see that $\phi\left(I_{4}\right)=I_{4}$. 
Proof of Theorem 3.1: For $n \geqslant 5$, the sufficiency part can be verified readily. For $n=4$, suppose $\phi\left(I_{4}\right)=I_{4}$ and $\phi$ permutes the sets $\left\{T_{12}, T_{34}\right\},\left\{T_{13}, T_{24}\right\}$, and $\left\{T_{14}, T_{23}\right\}$. Since every element in $\mathbf{S P}_{4} \backslash \mathbf{T}_{4}$ has the form $T_{p q}+T_{r s}-I_{n}$ where $\{p, q, r, s\}=\{1,2,3,4\}$, we have $\phi\left(T_{p q}+T_{r s}-I_{n}\right)=T_{i j}+T_{k l}-I_{n}$ where $\{i, j, k, l\}=\{1,2,3,4\}$. Thus, $\phi$ will map the set $\mathbf{S P}_{4} \backslash \mathbf{T}_{4}$ onto itself. So, $\phi\left(\mathbf{S P}_{n}\right)=\mathbf{S P}_{n}$, or equivalently, $\phi\left(\operatorname{conv} \mathbf{S P}_{n}\right)=$ conv $\mathbf{S P}_{n}$.

To prove the converse, assume that $\phi$ leaves invariant $\mathbf{S P}_{n}$ and conv $\mathbf{S P}_{n}$. Then $\phi$ is bijective. By Lemmas 3.4 and $2.4, \phi\left(I_{n}\right)=I_{n}$ and $\phi\left(\mathrm{T}_{n}\right)=\mathrm{T}_{n}$. Note that $T_{p q}, T_{r s} \in \mathrm{T}_{n}$ correspond to disjoint transpositions if and only if $T_{p q}+T_{r s}-I_{n} \in \mathbf{S P}_{n}$. Thus, $\phi$ maps pairs of disjoint transpositions to pairs of disjoint transpositions. Since $\phi$ is bijective, we are done if $n=4$.

Suppose $n \geqslant 5$. It suffices to show that $\phi$ can be converted to the identity mapping on $\mathrm{V}_{n}$ by the composite of a sequence of mappings of the form $X \mapsto P \phi(X) P^{t}$. In the following discussion, we say that two different transpositions $T_{i j}$ and $T_{k l}$ overlap if $\{i, j\} \cap\{k, l\}$ is a singleton, that is, $T_{i j}$ and $T_{k l}$ are not disjoint transpositions. Now, any two members in $\left\{T_{1 j}: 2 \leqslant j \leqslant n\right\}$ overlap, so the same must be true for $\left\{\phi\left(T_{1 j}\right)\right.$ : $2 \leqslant j \leqslant n\}$. Since $\phi\left(T_{12}\right)$ and $\phi\left(T_{13}\right)$ overlap, we can replace $\phi$ by a mapping of the form $X \mapsto P \phi(X) P^{t}$ so that $\phi(X)=X$ for $X=I_{n}, T_{12}$, and $T_{13}$. Since $\phi\left(T_{14}\right)=T_{p q}$ overlaps with each of $T_{12}$ and $T_{13}$, we see that $p=1$ or $(p, q)=(2,3)$. If the latter holds, then $\phi\left(T_{15}\right)=T_{r s}$ overlaps with each of $T_{12}, T_{13}$, and $T_{23}$, which is impossible. So, we have $\phi\left(T_{14}\right)=T_{1 s}$ for some $s \geqslant 4$. We may assume that $s=4$; otherwise, replace $\phi$ by the mapping $X \mapsto T_{4 s} \phi(X) T_{4 s}$. Repeating the same arguments, we may assume that $\phi\left(T_{1 j}\right)=T_{1 j}$ for $j=1, \ldots, n$. Now, $T_{23}$ is the only matrix in $\mathbf{T}_{n}$ that is disjoint with $T_{1 j}$ for all $j \geqslant 4$. So, $\phi\left(T_{23}\right)=T_{23}$. Similarly, we have $\phi\left(T_{2 j}\right)=T_{2 j}$ for all $j \geqslant 4$. Inductively, we see that $\phi\left(T_{r s}\right)=T_{r s}$ for all $r, s$. Since $\phi$ fixes every element in a spanning set for $\mathrm{V}_{n}$, the result follows.

\section{Symmetric Doubly Stochastic Matrices}

In this section, we give a different proof of the result in [5] concerning the characterisation of linear maps leaving invariant the sets $\mathbf{E}_{n}$ or conv $\mathbf{E}_{n}=\mathbf{S D S}_{n}$. Note that if $n=2$, then $\mathbf{E}_{n}$ reduces to $\mathbf{T}_{n}$. So, we assume that $n \geqslant 3$ in our consideration.

TheOREM 4.1. Suppose $n \geqslant 3$. Let $\mathcal{S}$ be $\mathbf{E}_{n}$ or conv $\mathbf{E}_{n}=\mathbf{S D S}_{n}$. A linear map $\phi: \mathrm{V}_{n} \rightarrow \mathrm{V}_{n}$ satisfies $\phi(\mathcal{S})=\mathcal{S}$ if and only if there is $P \in \mathbf{P}_{n}$ such that $\phi$ has the form $X \mapsto P^{t} X P$.

The sufficiency part of Theorem 4.1 can be verified readily. We divide the proof of the necessity part into several lemmas. Similar to the proofs in the previous section, a key step is to show that $\phi\left(I_{n}\right)=I_{n}$ (see Lemma 4.4).

To represent matrices in $\mathbf{E}_{n}$, we use the following notation. Each matrix in $\mathbf{E}_{n}$ will be represented formally as a product of 1-cycles, 2-cycles, and odd cycles of length at 
least 3. The presence of an 1-cycle $\left(i_{1}\right)$ in the product will mean that the matrix has 1 in the $\left(i_{1}, i_{1}\right)$ position; the presence of a 2-cycle $\left(i_{1}, i_{2}\right)$ will mean that the matrix has 1 in the $\left(i_{1}, i_{2}\right)$ and $\left(i_{2}, i_{1}\right)$ positions; the presence of a $k$-cycle $\left(i_{1}, i_{2}, i_{3}, \ldots, i_{k}\right)$, where $k$ is an odd integer $\geqslant 3$, will mean that the matrix has $1 / 2$ in the $\left(i_{1}, i_{k}\right)$ and $\left(i_{k}, i_{1}\right)$ positions as well as in the $\left(i_{j}, i_{j+1}\right)$ and $\left(i_{j+1}, i_{j}\right)$ positions for $j=1,2, \ldots, k-1$. For example, $A=(3)(1,2)(4,5,6) \in \mathrm{E}_{6}$ means that the matrix $A$ has 1 in the $(3,3),(1,2)$, and $(2,1)$ positions, has $1 / 2$ in the $(4,5),(4,6),(5,4),(5,6),(6,4)$, and $(6,5)$ positions, and has 0 in all other positions.

Lemma 4.2. Suppose $n \geqslant 3$. Let $C_{n}$ be the sum of matrices in $\mathbf{E}_{n}$. Then

$$
C_{n}=a_{n} I_{n}+b_{n} \widetilde{J}_{n}
$$

for some positive numbers $a_{n}$ and $b_{n}$.

(a) If $n \geqslant 3$, then $a_{n}<2 b_{n}$.

(b) Suppose $n \geqslant 3$ and $n \neq 4$, and let $A \in \mathbf{E}_{n}$. Then all nonzero entries of $A$ equal $1 / 2$, that is, $A$ can be represented as a product of odd cycles each of length at least 3 , if and only if

$$
\left(C_{n}-2 b_{n} A\right) /\left(a_{n}+(n-3) b_{n}\right) \in \mathbf{S D S}_{n} .
$$

Proof: For any $P \in \mathbf{P}_{n}$, clearly

$$
P^{t} C_{n} P=P^{t}\left(\sum_{A \in \mathrm{E}_{n}} A\right) P=\sum_{A \in \mathrm{E}_{n}} P^{t} A P=\sum_{A \in \mathbf{E}_{n}} A=C_{n} .
$$

By Lemma 2.3, $C_{n}=a_{n} I_{n}+b_{n} \widetilde{J}_{n}$ for some positive real numbers $a_{n}$ and $b_{n}$.

(a) Note that $a_{n}$ is equal to the number of matrices in $\mathbf{E}_{n}$ with 1 in the $(1,1)$ position. Thus, $a_{n}=\left|\mathbf{E}_{n-1}\right|$. Now, the common row sum of $C_{n}$ is $a_{n}+(n-1) b_{n}$, and since each member of $\mathbf{E}_{n}$ in the summation that produces $C_{n}$ contributes an increment of 1 to the row sum, it follows that

$$
\left|\mathbf{E}_{n}\right|=a_{n}+(n-1) b_{n}=\left|\mathbf{E}_{n-1}\right|+(n-1) b_{n} .
$$

Thus, $b_{n}=\left(\left|\mathbf{E}_{n}\right|-\left|\mathbf{E}_{n-1}\right|\right) /(n-1)$. So, $a_{n}<2 b_{n}$ if and only if

$$
\left|\mathbf{E}_{n-1}\right|<\frac{2\left(\left|\mathbf{E}_{n}\right|-\left|\mathbf{E}_{n-1}\right|\right)}{n-1},
$$

or equivalently,

$$
(n+1)\left|\mathbf{E}_{n-1}\right|<2\left|\mathbf{E}_{n}\right| .
$$

Let $2 \mathbf{E}_{n}$ be the multi-set (that is, a set with elements counting multiplicities) such that every matrix in $\mathbf{E}_{n}$ appears exactly twice in $2 \mathbf{E}_{n}$. (We sometimes refer to two 
identical matrices in $2 \mathbf{E}_{n}$ as two copies of a matrix of a given form.) To prove (4), it suffices to construct an $n+1$ element multi-set $S(X)$ in $2 \mathrm{E}_{n}$ for each matrix $X \in \mathrm{E}_{n-1}$ such that the union $\bigcup_{X \in E_{n-1}} S(X)$ (counting multiplicities) is a proper subset of $2 \mathrm{E}_{n}$ with $(n+1)\left|\mathbf{E}_{n-1}\right|$ elements.

To achieve our goal, for each $X \in \mathbf{E}_{n-1}$, we construct the $n+1$ element multi-set $S(X)$ as follows. First, we associate each $X \in \mathbf{E}_{n-1}$ with the two identical matrices

$$
X \oplus[n] \in 2 \mathbf{E}_{n} .
$$

To determine the other $n-1$ matrices in $S(X)$, we consider two types of matrices in $\mathbf{E}_{n-1}$.

TYPE 1. $A \in \mathbf{E}_{n-1}$ has nonzero diagonal entries at the $i_{1}, i_{2}, \ldots, i_{p}$ positions with 1 $\leqslant i_{1}<\cdots<i_{p} \leqslant n-1$ for some $p \geqslant 1$, that is, the cycle decomposition of $A$ includes the 1 -cycles $\left(i_{1}\right), \ldots,\left(i_{p}\right)$. For $r=1, \ldots, n-1$, we construct $\widetilde{A}_{r} \in 2 \mathrm{E}_{n}$ as follows.

(i) If $A$ has $(r)$ as an 1-cycle in the cycle decomposition, replace $(r)$ by the 2-cycle $(n, r)$ to obtain $\widetilde{A}_{r}$;

(ii) if $A$ has $(r, i)$ as a 2-cycle in the cycle decomposition, replace $(r, i)$ by the 3-cycle $(n, r, i)$ to obtain $\widetilde{A}_{r}$

(iii) if $A$ has $\left(r, j_{1}, \ldots, j_{k}\right)$ as an odd cycle with $k>1$ in the cycle decomposition, replace the cycles $\left(i_{1}\right)$ and $\left(r, j_{1}, \ldots, j_{k}\right)$ in $A$ by the single odd cycle $\left(i_{1}, n, r, j_{1}, \ldots, j_{k}\right)$ to obtain $\tilde{A}_{r}$. Here we always assume that $j_{1}<j_{k}$; otherwise, rewrite the cycle $\left(r, j_{1}, \ldots, j_{k}\right)$ as $\left(r, j_{k}, j_{k-1}, \ldots, j_{1}\right)$.

For every matrix $A \in \mathbf{E}_{n-1}$, the cycle decomposition of $\widetilde{A}_{r} \in 2 \mathbf{E}_{n}$ has $n$ in a cycle of length at least 2 , so $\widetilde{A}_{r}$ will not be of the form (5). Now, let $S(A)$ consist of $\widetilde{A}_{1}, \ldots, \widetilde{A}_{n-1}$ and the two identical matrices $A \oplus[n]$ in $2 \mathbf{E}_{n}$. If we consider the union of $S(A)$ for all Type 1 matrices $A \in \mathrm{E}_{n-1}$, we have the following observations.

1. The two identical matrices $\tilde{A} \in 2 \mathbf{E}_{n}$ of the form $\left(n, j_{1}, j_{2}\right)(\cdots)$ that contains at least one 1-cycle will be used in the construction of $S(A)$ for a unique Type 1 matrix $A$; namely, one copy of $\widetilde{A}$ will appear as $\widetilde{A}_{j_{1}}$ for $A=\left(j_{1}, j_{2}\right)(\cdots) \in \mathbf{E}_{n-1}$, and the other copy will appear as $\widetilde{A}_{j_{2}}$ for the same $A \in \mathbf{E}_{n-1}$.

2. Exactly one of the two identical matrices $\tilde{A} \in 2 \mathbf{E}_{n}$ of the form $\tilde{A}=(n, j)(\cdots)$ will be used in the construction of $S(A)$ for a unique Type 1 matrix $A$. In fact, the matrix $\tilde{A}$ will appear as $\widetilde{A}_{j}$ in $S(A)$ for $A=(j)(\cdots) \in \mathrm{E}_{n-1}$, that is, $A$ is obtained from $\tilde{A}$ by replacing $(n, j)$ with $(j)$.

3. At most one of the two identical matrices $\tilde{A} \in 2 \mathbf{E}_{n}$ of the form

$$
\widetilde{A}=\left(n, j_{2}, j_{3}, \ldots, j_{k-1}, j_{k}\right)(\cdots)
$$

with $k \geqslant 5$ will appear in $S(A)$ for a Type 1 matrix $A$, which is unique if it exists. In fact, by our construction, if $j_{3}<j_{k-1}$, then there exists $A_{1} \in \mathrm{E}_{n-1}$ such that $\tilde{A}$ 
$\in S\left(A_{1}\right)$ if and only if $A_{1}=\left(j_{k}\right)\left(j_{2}, j_{3}, \ldots, j_{k-1}\right)(\cdots)$ and $j_{k}<i_{p}$ for every 1-cycle $\left(i_{p}\right)$ in $\widetilde{A}$; if $j_{3}>j_{k-1}$, then there exists $A_{2} \in \mathrm{E}_{n-1}$ such that $\widetilde{A} \in S\left(A_{2}\right)$ if and only if $A_{2}$ $=\left(j_{2}\right)\left(j_{k}, j_{k-1}, \ldots, j_{3}\right)(\cdots)$ and $j_{2}<i_{p}$ for every 1-cycle $\left(i_{p}\right)$ in $\tilde{A}$.

TYPE 2. $B \in \mathbf{E}_{n-1}$ has no nonzero diagonal entries, that is, the cycle decomposition of $B$ has no 1-cycles. For $r=1, \ldots, n-1$, we construct the matrix $\widetilde{B}_{r}$ as follows.

First, in the cycle decomposition of $B \in \mathbf{E}_{n-1}$, replace the indices $1,2, \ldots, n-1$ respectively by $1,2, \ldots, r-1, r+1, \ldots, n$, and then construct $\widetilde{B}_{r}$ by inserting the 1-cycle $(r)$ into the resulting matrix. In the cycle decomposition of $\widetilde{B}_{r}$, if $n$ is contained in a 3-cycle, that is, $\widetilde{B}_{r}=(r)\left(n, j_{1}, j_{2}\right)(\cdots)$, then the two identical matrices $\widetilde{B}_{r} \in 2 \mathbf{E}_{n}$ were already used up in the construction of $S(A)$ for Type 1 matrices $A$; in such case, we modify $\widetilde{B}_{r}$ by changing $(r)\left(n, j_{1}, j_{2}\right)(\cdots)$ to $(n, r)\left(j_{1}\right)\left(j_{2}\right)(\cdots)$.

Since a Type 2 matrix does not have an 1-cycle, the above construction will not result in a matrix of the form $Y \oplus[n] \in 2 \mathrm{E}_{n}$. Let $S(B)$ consist of the matrices $\widetilde{B}_{1}, \ldots, \widetilde{B}_{n-1}$ and the two identical matrices $B \oplus[n] \in 2 \mathrm{E}_{n}$. If we consider the union of $S(B)$ for all Type 2 matrices $B \in \mathbf{E}_{n-1}$, we have the following observations:

1. Excluding matrices of the form (5), at most one of the two identical matrices in $2 \mathrm{E}_{n}$ of any given form will appear in the union of $S(B)$ for all Type 2 matrices $B$. Indeed, if $\widetilde{B} \in 2 \mathbf{E}_{n}$ has exactly one 1-cycle $(i)$ where $i<n$, then at most a single copy of $\widetilde{B}$ will appear in $S(B)$ for the unique Type 2 matrix $B \in \mathrm{E}_{n-1}$ formed by removing $(i)$ from the cycle decomposition of $\widetilde{B}$ and replacing the indices $1,2, \ldots, i-1, i+1, \ldots, n$ with $1,2, \ldots, n-1$, respectively.

If $\widetilde{B} \in 2 \mathbf{E}_{n}$ has exactly two 1-cycles and $n$ is contained in a 2-cycle, that is, $\widetilde{B}$ $=\left(j_{1}\right)\left(j_{2}\right)(n, i)(\cdots)$, then exactly one copy of $\widetilde{B}$ will appear in $S(B)$ for the unique Type 2 matrix $B$ formed by constructing the intermediary matrix $\left(n, j_{1}, j_{2}\right)(i)(\cdots)$, removing (i) from the cycle decomposition, and replacing the indices $1,2, \ldots, i-1, i+1, \ldots, n$ in the intermediary matrix with the indices $1,2, \ldots, n-1$, respectively. No other forms of $\widetilde{B} \in 2 \mathbf{E}_{n}$ will appear in $S(B)$ for a Type 2 matrix $B \in \mathbf{E}_{n-1}$.

2. The union of $S(B)$ for all Type 2 matrices $B \in \mathbf{E}_{n-1}$ will not contain any matrices of the form $\left(n, j_{1}, j_{2}\right)(\cdots) \in 2 \mathbf{E}_{n}$ and thus will not contain any matrices in $2 \mathbf{E}_{n}$ of which two copies were used up in the construction of $S(A)$ for Type 1 matrices $A$.

Thus, $\bigcup_{X \in \mathbb{E}_{n-1}} S(X)$ (counting multiplicities) is a subset of the multi-set $2 \mathbf{E}_{n}$. If $n \neq 4$, this union is a proper subset of $2 E_{n}$ because any matrix in $2 E_{n}$ with a cycle decomposition having $n$ in a 3-cycle and having no 1-cycles does not belong to $S(X)$ for any $X \in \mathrm{E}_{n-1}$. For $n=4$, since $C_{4}=5 I_{4}+3 \widetilde{J}_{4}$, we have $a_{4}<2 b_{4}$. This completes the proof of (a).

(b) $(\Leftarrow)$ If there is some nonzero entry of $A$ that is not $1 / 2$, then $A$ has an 1 in either a diagonal entry or an off-diagonal entry. If 1 is in the $(i, i)$ diagonal position of $A(i \leqslant n)$, then the $(i, i)$ position of $C_{n}-2 b_{n} A$ is $a_{n}-2 b_{n}<0$. If 1 is in the $(i, j)$ 
off-diagonal position of $A$, then the $(i, j)$ position of $C_{n}-2 b_{n} A$ is $b_{n}-2 b_{n}<0$. In either case,

$$
\left(C_{n}-2 b_{n} A\right) /\left(a_{n}+(n-3) b_{n}\right) \notin \operatorname{SDS}_{n} .
$$

$\Leftrightarrow)$ If every nonzero entry of $A \in \mathrm{E}_{n}$ is equal to $1 / 2$, then all the diagonal entries of $2 b_{n} A$ are 0 and all the off-diagonal entries of $2 b_{n} A$ are either 0 or $b_{n}$. Thus, $C_{n}-2 b_{n} A$ is a nonnegative matrix with a common row sum and column sum of $a_{n}+(n-1) b_{n}-2 b_{n}$ $=a_{n}+(n-3) b_{n}$, giving $\left(C_{n}-2 b_{n} A\right) /\left(a_{n}+(n-3) b_{n}\right) \in \mathbf{S D S}_{n}$.

Lemma 4.3. Suppose $n \geqslant 3$ and $n \neq 4$. Let $D_{n}$ be the sum of matrices $A$ in $\mathbf{E}_{n}$ such that all nonzero entries of $A$ equal $1 / 2$. Then $D_{n}=c_{n} \widetilde{J}_{n}$ for some positive number $c_{n}$.

Proof: Note that except for $n=4$, there are matrices in $\mathrm{E}_{n}$ with all nonzero entries equal to $1 / 2$. In fact, if $n$ is odd, then a matrix in $\mathbf{E}_{n}$ corresponding to the long cycle $(1,2, \ldots, n)$ will be such a matrix; if $n \geqslant 6$ is even, then there are matrices whose cycle decompositions consist of two odd cycles, each of length at least 3 .

It is easy to check that $P^{t} D_{n} P=D_{n}$ for any $P \in \mathrm{P}_{n}$. By Lemma 2.3, $D_{n}$ is a linear combination of $I_{n}$ and $\tilde{J}_{n}$. Now, each matrix in $\mathbf{E}_{n}$ with all nonzero entries equal to $1 / 2$ has all diagonal entries equal to 0 . So, $D_{n}=c_{n} \widetilde{J}_{n}$ for some positive number $c_{n}$.

Lemma 4.4. If $\phi: \mathrm{V}_{n} \rightarrow \mathrm{V}_{n}$ satisfies $\phi(\mathcal{S})=\mathcal{S}$, where $\mathcal{S}=\mathrm{E}_{n}$ or $\operatorname{SDS}_{n}$, then $\phi\left(I_{n}\right)=I_{n}$.

PROOF: Since $\phi$ maps a spanning set of $\mathrm{V}_{n}$ onto itself, $\phi$ is a bijective linear map on $\mathbf{V}_{n}$. Now, conv $\mathbf{E}_{n}=\operatorname{SDS}_{n}$. Thus, $\phi\left(\mathbf{E}_{n}\right)=\mathbf{E}_{n}$ if and only if $\phi\left(\mathbf{S D S}_{n}\right)=\operatorname{SDS}_{n}$.

Define $C_{n}$ and $D_{n}$ as in Lemmas 4.2 and 4.3. Since $\phi\left(\mathbf{E}_{n}\right)=\mathbf{E}_{n}$, we have $\phi\left(C_{n}\right)=C_{n}$. Suppose $n \neq 4$. By Lemma 4.2 (b), if $X \in \mathrm{E}_{n}$ has all nonzero entries equal to $1 / 2$, then so does $\phi(X)$. Thus, we have $\phi\left(D_{n}\right)=D_{n}$. Since $I_{n}$ is a linear combination of $C_{n}$ and $D_{n}$, we see that $\phi\left(I_{n}\right)=I_{n}$.

Suppose $n=4$. Then $C_{4}=5 I_{4}+3 \tilde{J}_{4}$. One easily checks that a matrix $A \in \mathrm{E}_{4}$ satisfies $\left(C_{4}-5 A\right) / 9 \in \mathrm{SDS}_{4}$ if and only if

$$
A \in \mathcal{B}=\left\{I_{4},(1)(2,3,4),(2)(1,3,4),(3)(1,2,4),(4)(1,2,3)\right\} .
$$

Let $G=\sum_{A \in \mathcal{B}} A=2 I_{4}+\widetilde{J}_{4}$. Then $\phi(B)=\mathcal{B}$ and $\phi(G)=G$. Since $I_{4}=3 G-C_{4}$, we have $\phi\left(I_{4}\right)=\phi\left(3 G-C_{4}\right)=3 \phi(G)-\phi\left(C_{4}\right)=3 G-C_{4}=I_{4}$.

Proof of THE NECESSITY PART OF TheOREM 4.1: By Lemmas 4.4 and 2.4, we may assume that $\phi\left(I_{n}\right)=I_{n}$ and $\phi\left(\mathbf{T}_{n}\right)=\mathbf{T}_{n}$. If $n=3$, one readily checks that there is $P \in \mathbf{P}_{3}$ such that $P \phi(X) P^{t}=X$ for all $X \in \mathbf{T}_{3} \backslash\left\{I_{3}\right\}$. Thus, $\phi$ has the asserted form. If $n \geqslant 5$, then one can use arguments similar to those in the proof of Theorem 3.1 to show that $\phi$ has the asserted form.

Suppose $n=4$. One can again use the arguments similar to those in the proof of Theorem 3.1 to show that pairs of disjoint transpositions are mapped to pairs of disjoint 
transpositions. We may assume that $\phi\left(T_{12}\right)=T_{12}$ and $\phi\left(T_{13}\right)=T_{13}$; otherwise, replace $\phi$ by a mapping of the form $X \mapsto P \phi(X) P^{t}$ for some suitable $P \in \mathbf{P}_{4}$. Since $T_{14}$ is not disjoint with $T_{12}$ and $T_{13}$, we have $\phi\left(T_{14}\right) \in\left\{T_{14}, T_{23}\right\}$. Let $A=T_{12}+T_{13}+T_{14}$. Then $X \in \mathbf{E}_{4}$ satisifes $(A-X) / 2 \in \mathbf{S D S}_{4}$ if and only if $X \in\left\{T_{12}, T_{13}, T_{14}\right\}$. Thus, there are exactly 3 elements $Y \in \mathbf{E}_{4}$ such that $(\phi(A)-Y) / 2 \in \mathbf{S D S}_{4}$. However, if $\phi\left(T_{14}\right)=T_{23}$, then $\phi(A)=T_{12}+T_{13}+T_{23}$, and $Y \in \mathbf{E}_{4}$ satisifes $(\phi(A)-Y) / 2 \in \mathbf{S D S}_{4}$ if and only if $Y=T_{12}, T_{13}, T_{23}$, or $(1,2,3)(4)$, which is a contradiction. Thus, $\phi\left(T_{14}\right)=T_{14}$. One can then use the arguments similar to those in the proof of Theorem 3.1 to conclude that $\phi$ has the asserted form.

We note that one can also prove the necessity part of Theorem 4.1 using the arguments in Assertions 3 and 4 from the proof of [4, Theorem 2.2].

\section{REFERENCES}

[1] H. Chiang and C.K. Li, 'Linear maps leaving the alternating group invariant', Linear Algebra Appl. 340 (2002), 69-80.

[2] M. Katz, 'On the extreme points of a certain convex polytope,', J. Combinatorial Theory 8 (1970), 417-423.

[3] M. Katz, 'On the extreme points of a set of sub-stochastic and symmetric matrices', $J$. Math. Anal. Appl. 37 (1972), 576-579.

[4] C.K. Li, B.S. Tam and N.K. Tsing, 'Linear maps preserving permutation and stochastic matrices', Linear Algebra Appl. 341 (2002), 5-22.

[5] S.H. Lin and B.S. Tam, 'Strong linear preservers of symmetric doubly stochastic or doubly substochastic matrices', (preprint).

Department of Mathematics

College of William and Mary

P.O.Box 8795

Williamsburg, VA 23187-8795

United States of America

e-mail: hschia@wmalumni.com

ckli@math.wm.edu 